\title{
Flegmasia cerulea dolens en paciente con carcinoma testicular
}

\author{
Chirivella González I, Teruel Casasús A, Magro Molina A, Insa Mollá A, Rodríguez Braun E, \\ Cervantes Ruipérez A. \\ Servicio de Hematología y Oncología Médica. Hospital Clínico Universitario de Valencia. \\ Universidad de Valencia. \\ Actas Urol Esp. 2006;30(10):1043-1045
}

\section{RESUMEN}

FLEGMASIA CERULEA DOLENS EN PACIENTE CON CARCINOMA TESTICULAR

Describimos un caso de flegmasia cerulea dolens secundaria a una trombosis venosa profunda por compresión de la vena cava inferior, en un varón de 31 años con un tumor germinal. Necesitó un tratamiento urgente con agentes fibrinoliticos, heparina intravenosa y quimioterapia. Con el tratamiento obtuvo una respuesta completa tumoral y una completa revascularización de la vena cava y vena femoral derecha.

Palabras clave: Flegmasia cerulea dolens. Trombosis venosa. Trombolisis. Tumor germinal.

\section{ABSTRACT}

\section{PHLEGMASIA CERULEA DOLENS IN PATIENTS WITH TESTICULAR CARCINOMA}

We describe a case of phlegmasia cerulea dolens secondary to venous thrombosis due to compression of inferior vena cava, in a 31-year-old man with a germ cell tumour. He was treated with systemic thrombolytic agents, intravenous heparin and urgent chemotherapy He presented a complete tumoral response and complete revascularization of the vena cava and right femoral vein.

Key words: Phlegmasia cerulea dolens. Venous thrombosis. Thrombolysis. Germ cell tumor.

$\mathrm{L}$ os tumores germinales pueden presentarse con adenopatías retroperitoneales voluminosas. Éstas pueden comprimir o infiltrar la vena cava inferior provocando una trombosis venosa profunda. Una complicación poco frecuente, pero posible, es la flegmasia cerulea dolens. Su rápido diagnóstico y tratamiento es fundamental para la vida del paciente y mantener sin alteraciones el sistema venoso en las piernas. Describimos un caso de flegmasia cerulea dolens, por una trombosis venosa profunda (TVP) en miembro inferior derecho, provocada por una metástasis retroperitoneal de un seminoma.

\section{CASO CLÍNICO}

Paciente de 35 años sin antecedentes patológicos de interés. Acudió a urgencias por presentar desde hacia dos semanas dolor lumbar y disnea progresiva. A la exploración física destacaba una tumoración dura y no dolorosa en el testículo derecho de $2 \mathrm{~cm}$. En la radiografía de tórax se observaban múltiples nódulos pulmonares metastásicos.

Ante la sospecha de tumor germinal testicular, se pautó analgesia y se solicitó estudio mediante marcadores tumorales y TAC tóraco-abdóminopélvico. Se programó orquiectomía para la semana siguiente.

Antes de la fecha de la intervención quirúrgica el paciente acudió a urgencias por presentar dolor y aumento del perímetro del miembro inferior derecho. A la exploración destacaba edema en toda la pierna derecha, signo de Hommans positivo, pulsos arteriales periféricos palpables y coloración normal.

En el TAC tóraco-abdómino-pélvico se observaban múltiples nódulos pulmonares bilaterales, adenopatías mediastínicas, y una masa adenopá- 
tica retroperitoneal que englobaba cava y aorta, colapsaba cava inferior y aorta, producía trombosis de cava y femoral derecha (Fig. 1). Los marcadores tumorales alfa-fetoproteína y HCG fueron normales, $\mathrm{LDH}$ aumentada $(2.900 \mathrm{mU} / \mathrm{ml})$.

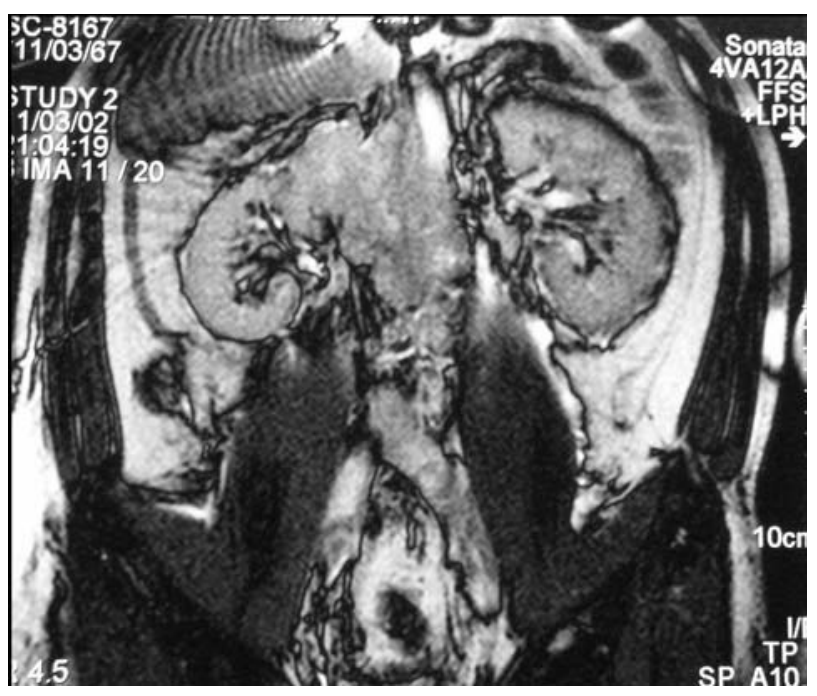

FIGURA 1

Ante la clínica de TVP de miembro inferior derecho, inició tratamiento con heparina de bajo peso molecular a dosis terapéuticas. Durante las horas siguientes presentó un rápido empeoramiento de la pierna con aumento del edema, dolor, y aparición de una coloración azulada, con signos claros de flegmasia cerulea dolens. Se decidió trombolisis con rtPA y anticoagulación con heparina sódica IV. La dosis que se administró de rTPA: $100 \mathrm{mg}$ a $50 \mathrm{ml} / \mathrm{h}$ a pasar en $2 \mathrm{~h}$. Se realizaron los siguientes controles analíticos pretrombolisis: hemoglobina ( $\mathrm{Hb})$, hematocrito (Hto), Dímero-D (D-D) y fibrinógeno e inmediatamente tras la misma con: Hb, Hto, D-D, fibrinógeno y TTPA. Si el TTPA es inferior a 80 segundos se inicia la perfusión de heparina sódica sin bolo (24.000 UI en 500 cc de suero fisiológico a 21 $\mathrm{ml} / \mathrm{h}$ ); si el TPTA es superior a 80 segundos ha de repetirse cada 2 horas hasta que sea inferior a 80 segundos para iniciar la perfusión de heparina. Tras finalizar la trombolisis presentó disminución del edema, desaparición de la coloración azulada de la pierna y del dolor.

$\mathrm{El}$ informe anatomopatológico de la orquiectomía fue de un seminoma testicular que respetaba la albugínea y el cordón espermático. Ante paciente con un tumor testicular seminomatoso avanzado con características de buen pronóstico, se pautó tratamiento quimioterápico con esquema BEP un total de 3 ciclos, y continuó tratamiento anticoagulante con warfarina.

Tras completar el tratamiento, la valoración fue de respuesta completa radiológica con normalización de LDH. Respecto a la pierna derecha, actualmente, presenta un ligero aumento del perímetro a nivel del muslo con circulación colateral, pero puede caminar con normalidad y sin dolor. Durante el seguimiento ha presentado varias progresiones de enfermedad que han respondido a tratamiento quimioterápico, pero no ha vuelto a presentar problemas en la pierna, y realiza una vida normal.

\section{DISCUSIÓN}

Los tumores germinales son curables en un alto porcentaje, por lo que es importante no retrasar el diagnóstico e iniciar lo antes posible el tratamiento, sobre todo en pacientes con situaciones críticas asociadas a la progresión del tumor. La detección rápida del tumor testicular es esencial y se ha demostrado que el retraso en más de 3 meses en el diagnóstico, se correlaciona con una disminución significativa de la supervivencia al año (94 vs 98\%) ${ }^{1}$.

La trombosis de la vena cava inferior y de la vena renal izquierda es una complicación poco frecuente del cáncer testicular. La causa más frecuente es la compresión extrínseca por adenopatías interaorto-cavas, pero también se ha descrito asociado a una coagulopatía intravascular diseminada de origen neoplásico (síndrome de Trosseaus) $)^{2}$.

La compresión o la invasión de la vena cava se produce con más frecuencia en los tumores testiculares derechos, ya que los ganglios afectos en este lado suelen ser interaórtico-cava a nivel de la arteria renal derecha. En algunos pacientes la primera manifestación es un tromboembolismo pulmonar, una trombosis venosa profunda o un sindrome de Budd-Chiari con un fallo hepatorenal. Revisando la literatura sólo hemos encontrado publicado un caso de flegmasia cerulea dolens en un paciente con cáncer testicular ${ }^{3}$.

Las consecuencias más importantes de la trombosis venosa profunda son el tromboembo- 
lismo pulmonar y la flegmasia cerulea dolens. En esta última la pierna, debido a la desoxigenación de la hemoglobina, toma una coloración azulada. La flegmasia cerulea dolens (PCD) produce una mortalidad del $25 \%$ con un $12-25 \%$ de riesgo de amputación de la pierna en los supervivientes ${ }^{4}$. Las complicaciones a largo plazo son la insuficiencia venosa profunda y la formación de venas varicosas.

La anticoagulación es el principal tratamiento para la TVP aguda. Los objetivos de la anticoagulación son la prevención de la extensión del trombo y de las recurrencias precoces o tardias de la TVP y del TEP.

La necesidad de anticoagulación en pacientes con TVP se basa en estudios realizados hace más de 40 años. La última conferencia de consenso sobre tratamiento antitrombótico y trombolítico, recomienda para el tratamiento de la TVP aguda la utilización conjunta de antagonistas de la vitamina-K y heparina (de bajo peso molecular o no fraccionada). Se recomienda el uso de la heparina al menos 5 días y su retirada cuando se alcance un INR estable $\geq 2,0^{5}$.

El valor relativo de la terapia trombolítica o anticoagulante para el tratamiento inicial de la TVP proximal, ha sido tema de discusión desde 1970. Hay seis estudios randomizados que comparan estreptoquinasa y heparina en el tratamiento inicial de la TVP aguda, utilizando la venografía como método diagnóstico y de valoración del tratamiento. El resultado de todos ellos mostró que la estreptoquinasa es 3,7 veces superior a la heparina para conseguir la trombolisis (con un intervalo de confianza del 95\% y unos límites entre 2,5 y 5,7). Sólo tres de esos 6 estudios permiten comparar la incidencia de sangrado mayor, que fue 2,9 veces superior en los grupos tratados con estreptoquinasa ${ }^{6}$. Resultados similares se han obtenido al utilizar uroquinasa y activador recombinante del plasminógeno (rtPA) como agentes fibrinoliticos.
Entonces, ¿cuándo se recomienda la utilización de fibrinolíticos en el tratamiento inicial de la TVP aguda?. La última conferencia de consenso recomienda el tratamiento inicial con agentes fibrinoliticos en pacientes con trombosis ileofemoral masiva con riesgo de gangrena secundaria a la oclusión 5 . En el caso que nos ocupa la obstrucción ileofemoral aguda y masiva, en progresión a pesar del tratamiento iniciado con heparina, estaba conduciendo a una gangrena inminente del miembro trombosado y en consecuencia, poniendo en riesgo la vida del paciente. De ahí la elección de la terapia trombolítica.

\section{REFERENCIAS}

1. Hernes EH, Harstad K, Fossa D. Changing incidence and delay of testicular cancer in southern Norway (1981-1992). Eur Urol. 1996;30(3):349-357.

2. Castiñeiras J, Cabello P, López A, Vilches J, RodríguezRubio F. Sindrome de Trousseas: trombosis de la vena cava inferior y de la vena renal izquierda asociada a una neoplasia testicular. Actas Urol. 1988;12(5):402-410.

3. Mulatero C, Brogan G, Oliver RT. Advanced testicular cancer presenting with phegmasia cerulea dolens. Postgrad Med J. 2000;76(894):234-236.

4. Weaver FA, Meacham PW, Adkins RB, Dean RH. Phlegmasia cerulea dolens: therapeutic consideration. South Med J. 1988;81(3):306-312.

5. Buller HR, Agnelli G, Hull RD, Hyers TM, Prins MH, Raskob GE. Antithrombotic therapy for venous thromboembolic disease: the Seventh Conference on Antithrombotic and Thrombolytic Therapy. Chest 2004;126(3Supple):401S$428 \mathrm{~S}$.

6. Lensing AW, Hirsh J. Rationale and results of thrombolytic therapy for deep vein thrombosis. In Bernstein E, editors. Vascular diagnosis. $4^{\text {th }}$ ed. St. Louis, Mosby 1994, pp 875879.

Dra. I. Chirivella González

E-mail: chirivella_isa@gva.es

(Trabajo recibido el 13 de julio 2006) 